\title{
Synthesis and Characterization of Metal Complexes Possessing the 5-(2-Pyridyl) Pyrazolate Ligands: The Observation of Remarkable Osmium-Induced Blue Phosphorescence in Solution at Room Temperature
}

\author{
Pei-Chi Wu, ${ }^{\dagger} \mathrm{J}$ en-Kan Yu, ${ }^{\ddagger}$ Yi-Hwa Song, ${ }^{\dagger}$ Yun Chi $^{*},{ }^{\dagger}+$ Pi-Tai Chou, ${ }^{*}, \neq$ \\ Shie-Ming Peng, ${ }^{\ddagger}$ and Gene-Hsiang Lee \\ Department of Chemistry, National Tsing Hua University, 300, Hsinchu, \\ Taiwan, Republic of China, and Department of Chemistry and Instrumentation Center, \\ National Taiwan University, 106, Taipe, Taiwan, Republic of China
}

Received J uly 13, 2003

\begin{abstract}
A total of three distinctive main group and transition metal complexes containing the 2-pyridyl pyrazolate (pypz) ligand were prepared, namely, $\left[\mathrm{B}\left(\mathrm{C}_{6} \mathrm{~F}_{5}\right)_{2}(\mathrm{pypz})\right](\mathbf{1}),\left[\mathrm{Ru}(\mathrm{CO})_{2^{-}}\right.$ (pypz) $)_{2}$ (2), and [Os(CO) 2 (pypz) 2 (3), where (pypz)H = 3-trifluoromethyl-5-(2-pyridyl)pyrazole. Single-crystal X-ray diffraction studies were carried out on complexes $\mathbf{2}$ and $\mathbf{3}$, revealing octahedral coordination geometry with two $\mathrm{CO}$ ligands located at cis dispositions. While the pypz ligand arrangement for complex $\mathbf{2}$ is in cis- $\left(\mathrm{N}_{\mathrm{py}}, \mathrm{N}_{\mathrm{py}}\right)$ and trans- $\left(\mathrm{N}_{\mathrm{pz}}, \mathrm{N}_{\mathrm{pz}}\right)$, complex $\mathbf{3}$ reveals a different configuration, cis- $\left(\mathrm{N}_{\mathrm{pz}}, \mathrm{N}_{\mathrm{pz}}\right)$ and trans- $\left(\mathrm{N}_{\mathrm{py}}, \mathrm{N}_{\mathrm{py}}\right)\left(\mathrm{N}_{\mathrm{py}}\right.$ for pyridine- $\mathrm{N}$ and $\mathrm{N}_{\mathrm{pz}}$ for pyrazolate donor sites). Similar to that of the in-situ-prepared pypz anion, the boron complex $\left[\mathrm{B}\left(\mathrm{C}_{6} \mathrm{~F}_{5}\right)_{2}(\mathrm{pypz})\right]$ (1) exhibits a strong emission centered at $380 \mathrm{~nm}$, which is unambiguously assigned to fluorescence derived from the $\mathrm{S}_{1}\left(\pi \pi^{*}\right) \rightarrow \mathrm{S}_{0}$ transition. In contrast to the nonluminescent behavior for Ru complex 2, the Os complex $\mathbf{3}$ displays unique, strong room-temperature phosphorescence, showing vibronic progressions at 430, 457, and $480 \mathrm{~nm}$. The remarkable differences in photophysical properties were rationalized by a combination of $\pi$-el ectron accepting CO ligand, relative pypz orientation, and heavy-atom-enhanced spinorbit coupling effects.
\end{abstract}

\section{Introduction}

Third-row transition metal complexes incorporating simple polypyridine ligands, ${ }^{1}$ such as $2,2^{\prime}$-bipyridine (bpy) or 1,10-phenanthroline (phen), and cyclometalated ligands, ${ }^{2}$ such as 2-phenylpyridine and benzoquinoline, have attracted a great deal of interest in recent years. Research in this area was principally motivated by the use of these complexes in the study of excited-state electron and energy transfer ${ }^{3}$ as well as the potential applications in the fabrication of organic light-emitting diodes (OLEDs). ${ }^{4}$ Isoelectronic transition metal ions such as $\operatorname{Re}(\mathrm{I}), \mathrm{Os}(\mathrm{II})$, and Ir(III), possessing a unique $\mathrm{d}^{6}$-electron configuration, are particularly attractive because of their strong metal-ligand interaction, long-

\footnotetext{
† National Tsing Hua University.
}

₹ National Taiwan University.

(1) (a) J uris, A.; Balzani, V.; Barigelletti, F .; Campagna, S.; Belser, P.; Von Zelewsky, A. Coord. Chem. Rev. 1988, 84, 85. (b) Constable, E. C.; Steel, P. J . Coord. Chem. Rev. 1989, 93, 205. (c) Balzani, V.; J uris, A. Coord. Chem. Rev. 2001, 211, 97. (d) Serroni, S.; Campagna, S.; Puntoriero, F.; Di Pietro, C.; McClenaghan, N. D.; Loiseau, F. Chem. Soc. Rev. 2001, 30, 367.

(2) (a) Beeby, A.; Bettington, S.; Samuel, I . D. W.; Wang, Z. J . Mater. Chem. 2003, 13, 80. (b) Colombo, M. G.; Guedel, H. U. Inorg. Chem. 1993, 32, 3081. (c) Lu, W.; Mi, B.-X.; Chan, M. C. W.; Hui, Z.; Zhu, N.; Lee, S.-T.; Che, C.-M. Chem. Commun. 2002, 206. (d) Song, D.; Wu, Q.; Hook, A.; Kozin, I.; Wang, S. Organometallics 2001, 20, 4683.

(3) (a) Treadway, J . A.; L oeb, B.; Lopez, R.; Anderson, P. A.; Keene, F. R.; Meyer, T. J . Inorg. Chem. 1996, 35, 2242. (b) Fleming, C. N.; Maxwell, K. A.; DeSimone, J . M.; Meyer, T.J .; Papanikolas, J . M. J . Am. Chem. Soc. 2001, 123, 10336. (c) Chardon-Noblat, S.; Deronzier A.; Hartl, F.; Van Slageren, J .; Mahabiersing, T. Eur. J . Inorg. Chem. 2001, 613. lived excited states, and high luminescence efficiencies, which significantly improve the likelihood of energy transfer prior to radiative or nonradiative relaxation. The strong spin-orbit coupling expected for these heavy metal ions, with atomic numbers $Z=75-77$, would lead to an efficient intersystem crossing from the singlet excited state to the triplet manifold. Furthermore, mixing singl et and triplet excited states via spin-orbit coupling, to a great extent, would also remove the spinforbidden nature of the $\mathrm{T}_{1} \rightarrow \mathrm{S}_{0}$ radiative relaxation, resulting in highly intense phosphorescent emission. On the basis of these concepts, polypyridine and cyclometalated types of ligands tend to form rigid molecular frameworks with the aforementioned metal ions and then give rise to desirable absorption and emission characteristics. More specifically, they normally display intense ligand-centered $\pi-\pi^{*}$ chromophores in the ultraviol et region and a weaker metal-to-ligand charge transfer (MLCT) transition in the lower energy, visible region. The strong absorption and emission characteristics derived from the $\pi-\pi^{*}$ and MLCT energy levels

(4) (a) Lamansky, S.; Djurovich, P.; Murphy, D.; Abdel-Razzaq, F.; Lee, H.-E.; Adachi, C.; Burrows, P. E.; Forrest, S. R.; Thompson, M. E. J . Am. Chem. Soc. 2001, 123, 4304. (b) Lamansky, S.; Djurovich, P.; Murphy, D.; Abdel-Razzaq, F.; Kwong, R.; Tsyba, I.; Bortz, M.; Mui, B.; Bau, R.; Thompson, M. E. Inorg. Chem. 2001, 40, 1704. (c) Brooks, J .; Babayan, Y.; Lamansky, S.; Djurovich, P. I.; Tsyba, I.; Bau, R.; Thompson, M. E. I norg. Chem. 2002, 41, 3055. (d) Carlson, B.; Phelan, G. D.; Kaminsky, W.; Dalton, L.; J iang, X. Z.; S., L.; J en, A. K.-Y. J . Am. Chem. Soc. 2002, 124, 14162. 
<smiles>Fc1c(F)c(F)c(B2N3C=CC=CC3c3cc(C(F)(F)F)nn32)c(F)c1F</smiles>

(1)

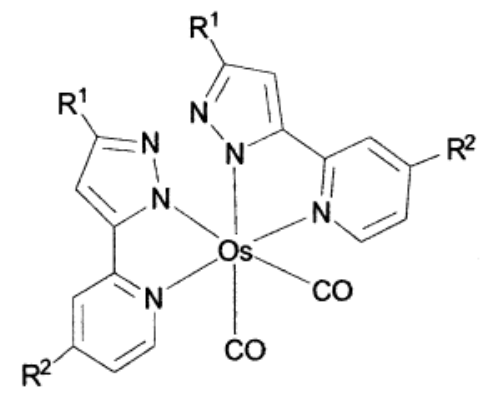

$$
\begin{aligned}
& \text { (3) } R^{1}=\mathrm{CF}_{3}, \mathrm{R}^{2}=\mathrm{H} \\
& \text { (4) } \mathrm{R}^{1}=\mathrm{C}_{2} \mathrm{~F}_{5}, \mathrm{R}^{2}=\mathrm{H} \\
& \text { (5) } \mathrm{R}^{1}=\mathrm{CF}_{3}, \mathrm{R}^{2}=\mathrm{Me}
\end{aligned}
$$

Figure 1. Structures of various $\mathrm{B}(\mathrm{III}), \mathrm{Ru}(\mathrm{II})$, and $\mathrm{Os}(\mathrm{II})$ complexes in this study.

make them suitable for use as the luminescent phosphors for future light-harvesting and OLED applications, respectively. ${ }^{5}$

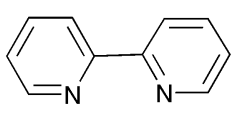

2,2'-bipyridine

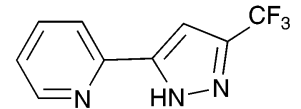

2-pyridyl pyrazole

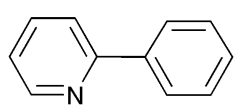

2-phenyl pyridine
In this paper, we wish to report the synthesis and characterization of a new series of 5-(2-pyridyl) pyrazolate (pypz) metal complexes with formula $\left[\mathrm{M}(\mathrm{CO})_{2-}\right.$ (pypz) $)_{2}$ ] where $\mathrm{M}=\mathrm{Ru}(\mathbf{2})$ or Os (3-5) (see Figure 1). The structural and chemical characteristics of the 2-pyridyl pyrazole (pypz)H are akin to those of 2,2'bipyridine and 2-phenylpyridine ligands in that 2-pyridyl pyrazole is capable of using two of its nitrogen atoms to generate a five-membered ring metal chelate interaction. ${ }^{6}$ Moreover, due to its strong acidity, ${ }^{7}$ the pyrazole site will readily lose a proton from the $\mathrm{NH}$ fragment to form a stable anionic ligand, subsequently producing neutral chelate complexes which, in sharp contrast to typical bipyridine ruthenium and osmium

(5) (a) Baldo, M. A.; Lamansky, S.; Burrows, P. E.; Thompson, M. E.; Forrest, S. R. Appl. Phys. Lett. 1999, 75, 4. (b) Wang, Y.; Herron, N.; Grushin, V. V.; LeCloux, D.; Petrov, V. Appl. Phys. Lett. 2001, 79, 449. (c) Adachi, C.; K wong, R. C.; Djurovich, P.; Adamovich, V.; Baldo, M. A.; Thompson, M. E.; Forrest, S. R. Appl. Phys. Lett. 2001, 79, 2082. (d) J iang, X.; J en, A. K.-Y.; Carlson, B.; Dalton, L. R. Appl. Phys. Lett. 2002, 80, 713.

(6) (a) J effery, J . C.; J ones, P. L.; Mann, K. L. V.; Psillakis, E.; McCleverty, J. A.; Ward, M. D.; White, C. M. Chem. Commun. 1997, 175. (b) Chadghan, A.; Pons, J .; Caubet, A.; Casabo, J .; Ros, J .; AlvarezLarena, A.; Piniella, J. F. Polyhedron 2000, 19, 855. (c) Pons, J.; Chadghan, A.; Casabo, J.; Alvarez-Larena, A.; Piniella, J. F.; Ros, J . Inorg. Chem. Commun. 2000, 3, 296.

(7) Tjiou, E. M.; Fruchier, A.; Pellegrin, V.; Tarrago, G. J . Heterocycl. Chem. 1989, 26, 893. complexes reported in the literature, have a high tendency to form ionic metal complexes. The strong $\sigma$-donor property of the pyrazolate, together with the $\pi$-accepting ability of the second pyridyl fragment, ${ }^{8}$ may provide a synergism of the electron delocalization so that the el ectron density is transferred from the pyrazolate to the metal ion and back to the pyridyl side of the ligand, enhancing the metal chelate interaction. For another comparison, although 2-phenylpyridine is effective in forming stable cycl ometalated compl exes with $\mathrm{Rh}(\mathrm{III}), \mathrm{Ir}(\mathrm{III})$, and $\mathrm{Pt}(\mathrm{II})$ ions,${ }^{9}$ it was rather inert to the $\mathrm{Ru}(\mathrm{II})$ and Os(II) metal ions and particularly, to our knowledge, failed to afford any Os(II) complexes. ${ }^{10}$ Alternatively, 2-pyridyl pyrazole is expected to extend the chemistry of 2-phenylpyridine, providing a great potential to isolate the respective $\mathrm{Ru}(\mathrm{II})$ and $\mathrm{Os}(\mathrm{II})$ complexes. On the basis of this strategic design, the resulting new series of 2-pyridyl pyrazoles incorporating $\mathrm{Os}(\mathrm{II})$ and $\mathrm{Ru}(\mathrm{II})$ complexes revealed remarkably different photophysical properties from previously reported anal ogues in that a strong ligand phosphorescence was resolved for $\left[\mathrm{Os}(\mathrm{CO})_{2}(\mathrm{pypz})_{2}\right]$ complexes in room-temperature solution phase. Detailed results and discussion are elaborated in the following sections.

\section{Experimental Section}

2.1. General Information and Materials. Mass spectra were obtained on a J EOL SX-102A instrument operating in electron impact (EI) mode or fast atom bombardment (FAB) mode. ${ }^{1} \mathrm{H}$ and ${ }^{13} \mathrm{C}$ NMR spectra were recorded on Varian Mercury-400 or INOVA-500 instruments; chemical shifts are quoted with respect to the internal standard tetramethylsilane for ${ }^{1} \mathrm{H}$ and ${ }^{13} \mathrm{C}$ NMR data. Elemental analyses were carried out at the NSC Regional Instrumentation Center at National Chao Tung University, Hsinchu, Taiwan. The pyrazole chelate ligands, 3-trifluoromethyl-5-(2-pyridyl)pyrazole, (pypz)H, 3-pentafluoroethyl-5-(2-pyridyl)pyrazole, (pypz2) H, and 3-trifluoromethyl-5-(4-methyl-2-pyridyl)pyrazole, (pypz3)H, were prepared according to the methods reported in the literature. ${ }^{11}$ All reactions were performed under a nitrogen atmosphere using anhydrous solvents or solvents treated with an appropriate drying reagent.

Synthesis of Complex 1. A $50 \mathrm{~mL}$ reaction flask was charged with $200 \mathrm{mg}$ of $\mathrm{B}\left(\mathrm{C}_{6} \mathrm{~F}_{5}\right)_{3}(0.38 \mathrm{mmol})$ and $30 \mathrm{~mL}$ of anhydrous THF solvent. To this solution was added $70 \mathrm{mg}$ of 3-trifluoromethyl-5-(2-pyridyl)pyrazole, (pypz)H (0.34 mmol), and the mixture was stirred at room temperature for $12 \mathrm{~h}$. After that, the solution was concentrated to dryness and the resulting oily residue was purified by recrystallization from a mixed solution of $\mathrm{CH}_{2} \mathrm{Cl}_{2}$ and methanol, giving a colorless crystalline solid, $\left[\mathrm{B}\left(\mathrm{C}_{6} \mathrm{~F}_{5}\right)_{2}(\mathrm{pypz})\right]$ (1) (90 mg, $\left.0.16 \mathrm{mmol}, 48 \%\right)$.

Spectral data of 1: MS (EI), $\mathrm{m} / \mathrm{z} 557, \mathrm{M}^{+} ; 538,(\mathrm{M}-\mathrm{F})^{+}$; 390, $\left(\mathrm{M}-\mathrm{C}_{6} \mathrm{~F}_{5}\right)^{+} .{ }^{1} \mathrm{H}$ NMR $\left(400 \mathrm{MHz}, \mathrm{CDCl}_{3}, 294 \mathrm{~K}\right): \delta 8.59$ $\left(\mathrm{dd},{ }^{3} \mathrm{Hн}_{\mathrm{H}}=6.0 \mathrm{~Hz}\right.$, 4 $\left.\mathrm{Hн}=1.2 \mathrm{~Hz}, \mathrm{lH}, \mathrm{CH}_{\mathrm{py}}\right), 8.28\left(\mathrm{ddd},{ }^{3} \mathrm{Hн}\right.$

(8) (a) Hage, R.; Haasnoot, J . G.; Reedijk, J .; Vos, J . G. Chemtracts: Inorg. Chem. 1992, 4, 75. (b) Hage, R.; Haasnoot, J. G.; Reedijk, J . Wang, R.; Vos, J. G. Inorg. Chem. 1991, 30, 3263.

(9) (a) Sprouse, S.; King, K. A.; Spellane, P. J .; Watts, R. J . J . Am. Chem. Soc. 1984, 106, 6647. (b) Chassot, L.; Von Zelewsky, A. Inorg Chem. 1987, 26, 2814. (c) Grushin, V. V.; Herron, N.; LeCloux, D. D.; Marshall, W. J.; Petrov, V. A.; Wang, Y. Chem. Commun. 2001, 1494.

(10) The 5-(2-pyridyl)phenyl ligand can coordinate to the Os atom forming a five-membered chelate interaction. However, its parent compound was not prepared from oxidative addition, but from the metal exchange involving bis(2-pyridyl)phenylmercury and OsHCl(CO)(PPh $)_{3}$; see: Clark, A. M.; Rickard, C. E. F.; Roper, W. R.; Wright, L. J . Organometallics 1999, 18, 2813.

(ii) (a) Thiel, W. R.; Eppinger, J. Chem. Eur. J . 1997, 3, 696. (b) Singh, S. P.; Kumar, D.; J ones, B. G.; Threadgill, M. D. J . Fluorine Chem. 1999, 94, 199. 


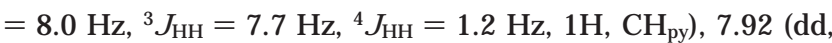
3) $\left.\mathrm{HH}=8.0 \mathrm{~Hz}, 4 \mathrm{~J} \mathrm{HH}=1.2 \mathrm{~Hz}, 1 \mathrm{H}, \mathrm{CH}_{\mathrm{py}}\right), 7.60\left(\mathrm{ddd},{ }^{3} \mathrm{~J} \mathrm{HH}=7.7\right.$

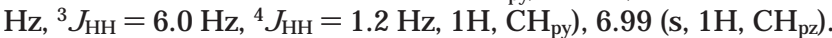
${ }^{19} \mathrm{~F}$ NMR $\left(470 \mathrm{MHz}, \mathrm{CDCl}_{3}\right): \delta-61.8\left(\mathrm{~s}, 3 \mathrm{~F}, \mathrm{CF}_{3}\right),-133.7(\mathrm{~s}$, 4F, CF ), -154.1 (s, 2F , CF), -161.9 (s, 4F, CF). Anal. Calcd for $\mathrm{C}_{21} \mathrm{H}_{5} \mathrm{BF}_{13} \mathrm{~N}_{3}: \mathrm{N}, 7.54 ; \mathrm{C}, 45.28 ; \mathrm{H}, 0.90$. Found: $\mathrm{N}, 7.41$; C, 45.21; $\mathrm{H}, 1.12$.

Synthesis of Complex 2. 3-Trifluoromethyl-5-(2-pyridyl)pyrazole, (pypz)H (620 mg, $2.91 \mathrm{mmol}), \mathrm{Ru}_{3}(\mathrm{CO})_{12}(300 \mathrm{mg}$ $0.47 \mathrm{mmol}$ ), and hexane solvent $(50 \mathrm{~mL})$ were added to a 160 $\mathrm{mL}$ stainless steel autodave. The autoclave was sealed and slowly brought up to $185^{\circ} \mathrm{C}$ for $36 \mathrm{~h}$. After that, the autoclave was cooled, the solvent was evaporated to dryness, and the solid residue was purified by column chromatography on $\mathrm{SiO}_{2}$, eluting with a 1:1 mixture of ethyl acetate and hexane. Only one major component was obtained. Removal of excess solvent produced a light yellow solid, which was purified by sublimation (150 mTorr/165 $\left.{ }^{\circ} \mathrm{C}\right)$, followed by crystallization from a mixture of $\mathrm{CH}_{2} \mathrm{Cl}_{2}$ /hexane, giving the ruthenium complex [Ru$(\mathrm{CO})_{2}(\mathrm{pypz})_{2}$ ] (2) as col orless rectangular crystals (191 mg, 0.33 $\mathrm{mmol}, 70 \%)$.

Spectral data of 2: MS (EI, $70 \mathrm{eV})$, observed $\mathrm{m} / \mathrm{z}$ (actual) [assignment] \{relative intensity\}: $582(582)\left[\mathrm{M}^{+}\right]\{2.88\}, 526$ (526) $\left[\mathrm{M}^{+}-2 \mathrm{CO}\right]\{12.5\} . \mathrm{IR}\left(\mathrm{CH}_{2} \mathrm{Cl}_{2}\right): v(\mathrm{CO}), 2076$ (s), 2017 (s) $\mathrm{cm}^{-1}$. ${ }^{1} \mathrm{H}$ NMR $\left(500 \mathrm{MHz}, \mathrm{d}_{6}\right.$-acetone, $\left.294 \mathrm{~K}\right): \delta$ 8.07-8.03 $\left(\mathrm{m}, 4 \mathrm{H}, \mathrm{H}_{\mathrm{py}}\right), 7.38\left(\mathrm{~d}, \mathrm{~J} \mathrm{Hн}=1 \mathrm{~Hz}, 2 \mathrm{H}, \mathrm{H}_{\mathrm{pz}}\right), 7.32$ (ddd, J нн $=$ 6 , 6, and $\left.3 \mathrm{~Hz}, 2 \mathrm{H}, \mathrm{H}_{\mathrm{py}}\right), 7.09$ (dd, J $\mathrm{HH}=6$ and $\left.1 \mathrm{~Hz}, 2 \mathrm{H}, \mathrm{H}_{\mathrm{py}}\right)$. ${ }^{13} \mathrm{C}$ NMR (125 M Hz, $\mathrm{d}_{6}$-acetone, $294 \mathrm{~K}$ ): $\delta 194.7$ (CO), 154.4 $\left(C_{p y}\right), 150.7\left(C_{p z}\right), 148.9\left(\mathrm{CH}_{p y}\right), 146.7\left(q,{ }^{2} \mathrm{~J} c F=36.6 \mathrm{~Hz}, \mathrm{C}_{\mathrm{pz}}\right)$, $141.7\left(\mathrm{CN}_{\mathrm{py}}\right), 124.5\left(\mathrm{CH}_{\mathrm{pz}}\right), 123.1\left(\mathrm{q},{ }^{1} \mathrm{~J} \mathrm{CF}=266.3 \mathrm{~Hz}, \mathrm{CF}_{3}\right)$, $121.7\left(\mathrm{CH}_{\text {py }}\right), 104.3\left(\mathrm{CH}_{\text {py }}\right) .{ }^{19} \mathrm{~F}$ NMR (470 MHz, d $\mathrm{d}_{6}$-acetone, $294 \mathrm{~K}$ ): $\delta-60.2$ (s). Anal. Calcd for $\mathrm{C}_{20} \mathrm{H}_{10} \mathrm{~F}_{6} \mathrm{~N}_{6} \mathrm{O}_{2} \mathrm{Ru}$ : $\mathrm{C}$ 41.372; N, 14.68; H, 1.85. Found: C, 41.32; N, 14.46; H, 1.73.

Synthesis of Complex 3. 3-Trifluoromethyl-5-(2-pyridyl)pyrazole (220 mg, $1.03 \mathrm{mmol}$ ) and finely pulverized $\mathrm{Os}_{3}(\mathrm{CO})_{12}$ (150 mg, $0.165 \mathrm{mmol}$ ) were loaded in a $20 \mathrm{~mL}$ Carius tube and degassed. This mixture/solution was then sealed under vacuum and placed in an oven maintaining temperatures of $180-185^{\circ} \mathrm{C}$ for 2.5 days, during which time its col or changed gradually from light yellow to red-brown and finally to orangeyellow. After the reaction was stopped, the tube was cooled and opened, and the contents were dissolved in acetone. The insoluble material was filtered off, the filtrate dried under vacuum, and the residue sublimed (300 mTorr/185 $\left.{ }^{\circ} \mathrm{C}\right)$. The product was then subjected to recrystal lization in $\mathrm{CH}_{2} \mathrm{Cl}_{2}$ and hexane, giving $\left[\mathrm{Os}(\mathrm{CO})_{2}(\mathrm{pypz})_{2}\right]$ (3) as colorless needlelike crystals (43 mg, $0.043 \mathrm{mmol}$ ) with a 39\% yield.

Spectral data of 3: MS (EI, $70 \mathrm{eV}$ ), observed m/z (actual) [assignment] \{relative intensity\}: $672(672)\left[\mathrm{M}^{+}\right]\{2.88\}, 616$ (616) $\left[\mathrm{M}^{+}-2 \mathrm{CO}\right]\{12.5\} . \mathrm{IR}\left(\mathrm{CH}_{2} \mathrm{Cl}_{2}\right): v(\mathrm{CO}), 2043$ (s), 1973 (s) $\mathrm{cm}^{-1} .{ }^{1} \mathrm{H}$ NMR $\left(400 \mathrm{MHz}, \mathrm{d}_{6}\right.$-acetone, $294 \mathrm{~K}$ ): $\delta 9.17$ (ddd, $\mathrm{J} н \mathrm{H}=6.0,1.5$, and $\left.1.0 \mathrm{~Hz}, 2 \mathrm{H}, \mathrm{H}_{\mathrm{py}}\right), 8.20$ (ddd, J ${ }_{\mu н}=8.0,8.0$, and $1.5 \mathrm{~Hz}, 2 \mathrm{H}, \mathrm{H}_{\mathrm{py}}$ ), 8.10 (ddd, J $\mathrm{HH}=8.0,1.5$, and $1.0 \mathrm{~Hz}$, $\left.2 \mathrm{H}, \mathrm{H}_{\mathrm{py}}\right), 7.48$ (ddd, J нн $=8.0,6.0$, and $\left.1.5 \mathrm{~Hz}, 2 \mathrm{H}, \mathrm{H}_{\mathrm{py}}\right), 7.10$ $\left(\mathrm{s}, 2 \mathrm{H}, \mathrm{H}_{\mathrm{pz}}\right) .{ }^{13} \mathrm{C}$ NMR (125 MHz, $\mathrm{d}_{6}$-acetone, $\left.294 \mathrm{~K}\right): \delta 177.6$ (CO), $157.1\left(\mathrm{CN}_{\mathrm{py}}\right), 155.8\left(\mathrm{C}_{\mathrm{py}}\right), 151.7\left(\mathrm{C}_{\mathrm{pz}}\right), 144.1\left(\mathrm{q},{ }^{2} \mathrm{~J} \mathrm{cF}=35.5\right.$ $\left.\mathrm{Hz}, \mathrm{C}_{\mathrm{pz}}\right), 141.3\left(\mathrm{CH}_{\mathrm{py}}\right), 125.2\left(\mathrm{CH}_{\mathrm{py}}\right), 123.1\left(\mathrm{q},{ }^{1} \mathrm{~J} \mathrm{CF}=265.7 \mathrm{~Hz}\right.$, $\left.\mathrm{CF}_{3}\right), 121.6\left(\mathrm{CH}_{\mathrm{py}}\right), 103.4\left(\mathrm{CH}_{\mathrm{pz}}\right) .{ }^{19} \mathrm{~F} \mathrm{NMR}\left(470 \mathrm{MHz}, \mathrm{d}_{6}-\right.$ acetone, $294 \mathrm{~K}$ ): $\delta-60.2$ (s). Anal. Calcd for $\mathrm{C}_{20} \mathrm{H}_{10} \mathrm{~F}_{6} \mathrm{~N}_{6} \mathrm{O}_{2-}$ Os: C, 35.82; N, 12.53; H, 1.50. Found: C, 35.67; N, 12.84; H, 1.78 .

Synthesis of Complex 4. The procedures were identical to those of complex 3, with the exception that $150 \mathrm{mg}$ of $\mathrm{Os}_{3^{-}}$ $(\mathrm{CO})_{12}(0.165 \mathrm{mmol})$ and $270 \mathrm{mg}$ of 3-pentafluoroethyl-5-(2pyridyl)pyrazole (pypz2)H (1.03 mmol) were used. After being heated for 3 days, the crude product was purified by sublimation $\left(0.38 \mathrm{Torr}, 210^{\circ} \mathrm{C}\right)$ and recrystallization from $\mathrm{CH}_{2} \mathrm{Cl}_{2}$ and hexane, giving a colorless crystalline solid, [Os(CO) $\left.)_{2}\left(p_{1} p z 2\right)_{2}\right]$ (4, $55 \mathrm{mg}, 0.071 \mathrm{mmol})$, with a $43 \%$ yield.

Spectral data of 4: MS (EI, $70 \mathrm{eV})$, observed m/z (actual) [assignment] \{relative intensity\}: $772(772)\left[\mathrm{M}^{+}\right]\{1.96\}, 716$
(716) $\left[\mathrm{M}^{+}-2 \mathrm{CO}\right]\{9.74\}$. IR $\left(\mathrm{CH}_{2} \mathrm{Cl}_{2}\right): v(\mathrm{CO}), 2043$ (s), 1973 (s) $\mathrm{cm}^{-1} .{ }^{1} \mathrm{H}$ NMR $\left(500 \mathrm{MHz}, \mathrm{d}_{6}\right.$-acetone, $294 \mathrm{~K}$ ): $\delta 9.17$ (ddd, $\mathrm{J}_{н н}=6.0,1.5$, and $\left.1.0 \mathrm{~Hz}, \mathrm{H}_{\mathrm{py}}\right), 8.19$ (ddd, $\mathrm{J}_{н н}=8.0,8.0$, and $1.5 \mathrm{~Hz}, \mathrm{H}_{\mathrm{py}}$ ), 8.11 (ddd, J нH $=8.0,1.5$, and $1.0 \mathrm{~Hz}, 2 \mathrm{H}, \mathrm{H}_{\mathrm{py}}$ ), 7.47 (ddd, J нн $=8.0,6.0$, and $\left.1.5 \mathrm{~Hz}, 2 \mathrm{H}, \mathrm{H}_{\text {py }}\right), 7.08(\mathrm{~s}, 2 \mathrm{H}$, $\left.\mathrm{H}_{\mathrm{pz}}\right) .{ }^{13} \mathrm{C}$ NMR (125 MHz, $\mathrm{d}_{6}$-acetone, $294 \mathrm{~K}$ ): $\delta 177.8$ (CO), $157.1\left(\mathrm{CN}_{\mathrm{py}}\right), 155.9\left(\mathrm{C}_{\mathrm{py}}\right), 152.0\left(\mathrm{C}_{\mathrm{pz}}\right), 142.4\left(\mathrm{t},{ }^{2} \mathrm{~J} \mathrm{cF}=27.2 \mathrm{~Hz}\right.$, $\left.\mathrm{C}_{\mathrm{pz}}\right), 141.2\left(\mathrm{CH}_{\mathrm{py}}\right), 125.1\left(\mathrm{CH}_{\mathrm{py}}\right), 121.6\left(\mathrm{CH}_{\mathrm{py}}\right), 119.9$ (qt, 1J $\mathrm{CF}$ $\left.=283.5 \mathrm{~Hz},{ }^{2} \mathrm{~J} \mathrm{CF}=38.9 \mathrm{~Hz}, \mathrm{CF}_{3}\right), 112.4\left(\mathrm{tq}^{1}{ }^{1} \mathrm{~J} \mathrm{CF}=247.0 \mathrm{~Hz}\right.$, 2] $\left.\mathrm{CF}=38.3 \mathrm{~Hz}, \mathrm{CF}_{2}\right), 104.3\left(\mathrm{CH}_{\mathrm{pz}}\right) .{ }^{19} \mathrm{~F} \mathrm{NMR}\left(470 \mathrm{MHz}, \mathrm{d}_{6}\right.$ acetone, $294 \mathrm{~K}): \delta-84.4\left(\mathrm{~s}, \mathrm{CF}_{3}\right),-109.1\left(\mathrm{~d},{ }^{3} \mathrm{FF}_{\mathrm{FF}}=276.8 \mathrm{~Hz}\right.$, $C F),-111.4\left(d, 3_{F F}=276.8 \mathrm{~Hz}, C F\right)$. Anal. Calcd for $\mathrm{C}_{22} \mathrm{H}_{10} \mathrm{~F}_{10} \mathrm{~N}_{6} \mathrm{O}_{2} \mathrm{Os}$ : C, 34.31; N, 11.07; $\mathrm{H}, 1.51$. Found: $\mathrm{C}, 34.29$; $\mathrm{N}, 10.91 ; \mathrm{H}, 1.31$.

Synthesis of 5. In a fashion similar to the synthesis of complex 3, $150 \mathrm{mg}$ of $\mathrm{Os}_{3}(\mathrm{CO})_{12}(0.165 \mathrm{mmol})$ and $240 \mathrm{mg}$ of 3-trifluoromethyl-5-(4-methyl-2-pyridyl)pyrazole, (pypz3)H (1.04 $\mathrm{mmol})$, were used. After being heated for 4 days, the crude product was purified by sublimation $\left(0.24 \mathrm{Torr}, 220^{\circ} \mathrm{C}\right)$, followed by recrystallization with $\mathrm{CH}_{2} \mathrm{Cl}_{2}$ and hexane, giving a colorless crystalline solid [Os(CO) $\left.)_{2}(\mathrm{pypz} 3)_{2}\right](5,34 \mathrm{mg}, 0.048$ $\mathrm{mmol}$ ) with a $29 \%$ yield.

Spectral data of 5: MS (EI, $70 \mathrm{eV}$ ), observed $\mathrm{m} / \mathrm{z}$ (actual) [assignment] \{relative intensity\}: $700(700)\left[\mathrm{M}^{+}\right]\{3.33\}, 644$ (644) $\left[\mathrm{M}^{+}-2 \mathrm{CO}\right]\{14.9\}$. IR $\left(\mathrm{CH}_{2} \mathrm{Cl}_{2}\right): v(\mathrm{CO}), 2041$ (s), 1970 (s) $\mathrm{cm}^{-1} .{ }^{1} \mathrm{H}$ NMR $\left(400 \mathrm{MHz}, \mathrm{d}_{6}\right.$-acetone, $294 \mathrm{~K}$ ): $\delta 8.97(\mathrm{~d}$, $\left.\mathrm{J} \mathrm{HH}=6.0 \mathrm{~Hz}, 2 \mathrm{H}, \mathrm{H}_{\mathrm{py}}\right), 7.95\left(\mathrm{~s}, 2 \mathrm{H}, \mathrm{H}_{\mathrm{py}}\right), 7.31(\mathrm{~d}, \mathrm{~J} \mathrm{HH}=6.0$ $\left.\mathrm{Hz}, 2 \mathrm{H}, \mathrm{H}_{\mathrm{py}}\right), 7.06\left(\mathrm{~s}, 2 \mathrm{H}, \mathrm{H}_{\mathrm{pz}}\right), 2.58$ (s, 6H, Me). ${ }^{13} \mathrm{C} \mathrm{NMR}(125$ $\mathrm{MHz}, \mathrm{d}_{6}$-acetone, $\left.294 \mathrm{~K}\right): \delta 177.8(\mathrm{CO}), 156.2\left(\mathrm{CN}_{\mathrm{py}}\right), 155.2$ $\left(C_{p y}\right), 153.7\left(C_{p y}\right), 151.8\left(C_{p z}\right), 144.0\left(q,{ }^{2}{ }_{C F}=35.4 \mathrm{~Hz}, C_{p z}\right)$, $126.2\left(\mathrm{CH}_{\mathrm{py}}\right), 122.3\left(\mathrm{q},{ }^{1} \mathrm{~J} \mathrm{CF}=241.8 \mathrm{~Hz}, \mathrm{CF}_{3}\right), 122.1\left(\mathrm{CH}_{\mathrm{py}}\right)$, 103.1 $\left(\mathrm{CH}_{\mathrm{pz}}\right), 21.2(\mathrm{Me}) .{ }^{19} \mathrm{~F}$ NMR $\left(470 \mathrm{MHz}, \mathrm{d}_{6}\right.$-acetone, 294 K): $\delta-59.8(\mathrm{~s})$. Anal. Calcd for $\mathrm{C}_{22} \mathrm{H}_{14} \mathrm{~F}_{6} \mathrm{~N}_{6} \mathrm{O}_{2} \mathrm{Os:} \mathrm{C}, 37.82 ; \mathrm{N}$ 12.03; H, 2.02. Found: C, 37.69; N, 12.01; H 2.08.

2.2. Measurements. Single-crystal $X$-ray diffraction data were measured on a Nonius Kappa or a Bruker SMART CCD diffractometer using $\lambda(\mathrm{M} \circ \mathrm{K} \alpha)$ radiation $(\lambda=0.71073 \AA)$. The data collection was executed using the SMART program. Cell refinement and data reduction were made with the SAINT program. The structure was determined using the SHELXTL/ PC program and refined using full-matrix least squares. All non-hydrogen atoms were refined anisotropically, whereas hydrogen atoms were placed at the calculated positions and included in the final stage of refinements with fixed parameters. Crystallographic refinement parameters of complexes $\mathbf{2}$ and $\mathbf{3}$ are summarized in Table 1, and the selective bond distances and angles of these complexes are listed in Tables 2 and 3 , respectively.

Steady-state absorption and emission spectra were recorded with a Hitachi (U-3310) spectrophotometer and an Edinburgh (FS920) fluorimeter, respectively. Nanosecond-microsecond lifetime studies were performed with an Edinburgh FL 900 photon-counting system with a hydrogen-filled lamp or a nitrogen lamp as the excitation source. The emission decays were analyzed by the sum of exponential functions, which allows partial elimination of instrument time broadening and thus renders a temporal resolution of $\sim 200$ ps. Occasionally, for the long-lived $(\gg \mu \mathrm{s})$ emission species the lifetime was measured with the laser photolysis technique in which the third or fourth harmonic of an Nd:YAG Iaser (8 ns, Continuum Surlite II) was used as the excitation source, coupled with a fast response photomultiplier (Hamamatsu model R5509-72) operated at $-80^{\circ} \mathrm{C}$. Typically, an average of 512 shots were acquired for each measurement. The transient absorption signal was recorded by a laser flash photolysis system (E dinburgh LP920), in which an Nd:YAG laser (355 nm) pumped optical parametric oscillator and a whitelight square pulse were used as pump and probe beams, respectively. The temporal resolution was limited by the excitation pulse duration of $\sim 8$ ns. 
Table 1. Crystal Data and Structure Refinement Parameters for Complexes 2 and 3

\begin{tabular}{|c|c|c|}
\hline & 2 & 3 \\
\hline $\begin{array}{l}\text { empirical formula } \\
\text { fw } \\
\text { diffractometer } \\
\text { temperature } \\
\text { cryst syst } \\
\text { space group } \\
\text { a } \\
\text { b } \\
\text { c } \\
\beta \\
\text { volume, Z } \\
\text { density (calcd) } \\
\text { abs coeff } \\
\text { F (000) } \\
\text { cryst size (mm³) } \\
\theta \text { ranges } \\
\text { no. of reflns collected } \\
\text { no. of indep reflns } \\
\text { no. of data/restraints/params } \\
\text { goodness-of-fit on } \mathrm{F}^{2} \\
\text { final } R \text { indices [l > 2 } \sigma(I)] \\
R \text { indices (all data) } \\
\text { largest diff peak and hole }\end{array}$ & $\begin{array}{l}\mathrm{C}_{20} \mathrm{H}_{10} \mathrm{~F}_{6} \mathrm{~N}_{6} \mathrm{O}_{2} \mathrm{Ru} \\
581.41 \\
\mathrm{~N} \text { onius KappaCCD } \\
295(2) \mathrm{K} \\
\text { orthorhombic } \\
\text { Pbca } \\
17.3441(2) \AA \\
13.4557(1) \AA \\
18.4594(2) \AA \\
4308.00(8) \AA^{3}, 8 \\
1.793 \mathrm{Mg}^{3} \mathrm{~m}^{3} \\
0.811 \mathrm{~mm}^{-1} \\
2288 \\
0.40 \times 0.40 \times 0.30 \\
2.21 \text { to } 27.50^{\circ} \\
22777 \\
4940[\mathrm{R}(\mathrm{int})=0.0483] \\
4940 / 0 / 317 \\
1.063 \\
\mathrm{R}_{1}=0.0456, \mathrm{wR}_{2}=0.1212 \\
\mathrm{R}_{1}=0.0825, \mathrm{wR}_{2}=0.1403 \\
1.163 \text { and }-0.686 \text { e } \AA^{-3}\end{array}$ & $\begin{array}{l}\mathrm{C}_{20} \mathrm{H}_{10} \mathrm{~F}_{6} \mathrm{~N}_{6} \mathrm{O}_{2} \mathrm{Os} \\
670.54 \\
\text { Bruker SMART APEX } \\
295(2) \mathrm{K} \\
\text { monoclinic } \\
\mathrm{P} 2 / \mathrm{c} \\
13.2724(7) \AA \\
13.5524(7) \AA \\
12.9716(7) \AA \\
109.229(1)^{\circ} \\
2203.1(2) \AA^{3}, 4 \\
2.022 \mathrm{Mg} / \mathrm{m}^{3} \\
5.870 \mathrm{~mm}^{-1} \\
1272 \\
0.28 \times 0.15 \times 0.07 \\
2.21 \text { to } 27.50^{\circ} \\
17612 \\
5072[\mathrm{R}(\mathrm{int})=0.0290] \\
5072 / 0 / 372 \\
1.026 \\
\mathrm{R}_{1}=0.0240, \mathrm{wR}_{2}=0.0550 \\
\mathrm{R}_{1}=0.0307, \mathrm{wR}_{2}=0.0582 \\
1.160 \text { and }-0.694 \mathrm{e} \AA^{-3}\end{array}$ \\
\hline
\end{tabular}

Table 2. Selected Bond Lengths $[\AA]$ ] and Angles [deg] for Complex 2

\begin{tabular}{lrlr}
\hline $\mathrm{Ru}-\mathrm{N}(1)$ & $2.136(3)$ & $\mathrm{Ru}-\mathrm{N}(2)$ & $2.058(3)$ \\
$\mathrm{Ru}-\mathrm{N}(4)$ & $2.140(3)$ & $\mathrm{Ru}-\mathrm{N}(5)$ & $2.047(3)$ \\
$\mathrm{Ru}-\mathrm{C}(1)$ & $1.892(4)$ & $\mathrm{Ru}-\mathrm{C}(2)$ & $1.876(5)$ \\
$\mathrm{O}(1)-\mathrm{C}(1)$ & $1.121(5)$ & $\mathrm{O}(2)-\mathrm{C}(2)$ & $1.128(5)$ \\
$\mathrm{N}(2)-\mathrm{N}(3)$ & $1.344(4)$ & $\mathrm{N}(5)-\mathrm{N}(6)$ & $1.351(4)$ \\
& & & \\
$\angle \mathrm{N}(1)-\mathrm{Ru}-\mathrm{C}(1)$ & $174.4(1)$ & $\angle \mathrm{N}(4)-\mathrm{Ru}-\mathrm{C}(2)$ & $173.6(2)$ \\
$\angle \mathrm{N}(1)-\mathrm{Ru}-\mathrm{N}(2)$ & $77.5(1)$ & $\angle \mathrm{N}(4)-\mathrm{Ru}-\mathrm{N}(5)$ & $77.1(1)$ \\
$\angle \mathrm{N}(2)-\mathrm{Ru}-\mathrm{N}(5)$ & $163.7(1)$ & $\angle \mathrm{C}(1)-\mathrm{Ru}-\mathrm{C}(2)$ & $91.8(2)$
\end{tabular}

Table 3. Selected Bond Lengths $[\AA]$ and Angles [deg] for Complex 3

\begin{tabular}{lrlr}
\hline $\mathrm{Os}(1)-\mathrm{N}(1)$ & $2.013(3)$ & $\mathrm{Os}(1)-\mathrm{N}(2)$ & $2.087(3)$ \\
$\mathrm{Os}(1)-\mathrm{C}(1)$ & $1.881(3)$ & $\mathrm{O}(1)-\mathrm{C}(1)$ & $1.136(4)$ \\
$\mathrm{N}(2)-\mathrm{N}(3)$ & $1.342(4)$ & $\mathrm{N}(1)-\mathrm{C}(6)$ & $1.364(4)$ \\
$\mathrm{N}(2)-\mathrm{C}(7)$ & $1.354(4)$ & $\mathrm{N}(3)-\mathrm{C}(9)$ & $1.350(4)$ \\
$\mathrm{C}(6)-\mathrm{C}(7)$ & $1.442(5)$ & $\mathrm{C}(7)-\mathrm{C}(8)$ & $1.383(5)$ \\
$\mathrm{C}(8)-\mathrm{C}(9)$ & $1.388(5)$ & & \\
$\angle \mathrm{N}(1)-\mathrm{Os}(1)-\mathrm{N}(2)$ & $77.3(1)$ & $\angle \mathrm{N}(1)-\mathrm{Os}(1)-\mathrm{N}(1 \mathrm{~A})$ & $169.0(1)$ \\
$\angle \mathrm{N}(1)-\mathrm{Os}(1)-\mathrm{N}(2 \mathrm{~A})$ & $94.7(1)$ & $\angle \mathrm{N}(2)-\mathrm{Os}(1)-\mathrm{C}(1)$ & $92.6(1)$ \\
$\angle \mathrm{N}(2)-\mathrm{Os}(1)-\mathrm{C}(1 \mathrm{~A})$ & $173.2(1)$ & &
\end{tabular}

\section{Results}

3.1. Preparation and Spectroscopic Characterization. 2-Pyridyl pyrazole, (pypz)H, can readily react with various metal source reagents, affording metal chelate complexes in high yields. The first example is represented by the direct treatment of $\mathrm{B}\left(\mathrm{C}_{6} \mathrm{~F}_{5}\right)_{3}$ and (pypz) $\mathrm{H}$ at room temperature, giving a tetrahedrally coordinated boron complex, $\left[\mathrm{B}\left(\mathrm{C}_{6} \mathrm{~F}_{5}\right)_{2}(\mathrm{pypz})\right]$ (1) (eq 1).

$$
\mathrm{B}\left(\mathrm{C}_{6} \mathrm{~F}_{5}\right)_{3}+(\mathrm{pypz}) \mathrm{H} \rightarrow\left[\mathrm{B}\left(\mathrm{C}_{6} \mathrm{~F}_{5}\right)_{2}(\mathrm{pypz})\right](\mathbf{1})+\mathrm{C}_{6} \mathrm{~F}_{5} \mathrm{H}
$$

Characterization of this boron complex was achieved using the routine spectroscopic methods as well as the previously determined crystal structural data of the phenyl-substituted boron complex $\left[\mathrm{B}\left(\mathrm{C}_{6} \mathrm{H}_{5}\right)_{2}(\mathrm{pypz})\right] .12 \mathrm{It}$ was found that the pyridyl nitrogen atom $\left(\mathrm{N}_{\mathrm{py}}\right)$ and the nearby nitrogen atom of the pyrazolate were both

(12) Cheng, C.-C.; Chou, P.-T.; Peng, S.-M.; Lee, G.-H.; Wu, P.-C.; Song, Y.-H.; Chi, Y. Chem. Commun. 2003, 2628. coordinated to the boron atom, forming a five-membered $\mathrm{C}_{2} \mathrm{~N}_{2} \mathrm{~B}$ chelate interaction. A similar bonding arrangement was observed in other tetrahedrally arranged $\mathrm{BPh}_{2}$ complexes with chelating ligands, such as (2pyridyl)-7-azaindole and (2-pyridyl)-7-indol e. ${ }^{13}$

A similar straightforward route was applied to the synthesis of the transition metal carbonyl complexes involving Ru(II) and Os(II) cations. For the complex [Ru$\left.(\mathrm{CO})_{2}(\mathrm{pypz})_{2}\right]$ (2), the reaction was best carried out in hexane, using a stainless steel autoclave to raise the reaction temperature to $185^{\circ} \mathrm{C}$. It is possible that the reaction proceeds in multistep processes involving prior cluster fragmentation ${ }^{14}$ to afford mononuclear intermediates such as $\operatorname{Ru}(\mathrm{CO})_{x}, 4 \leq x \leq 2$, together with pyrazole addition, $\mathrm{H}_{2}$ elimination, and $\mathrm{CO}$ displacement. The overall transformation is thus depicted as

$$
\begin{aligned}
& \mathrm{Ru}_{3}(\mathrm{CO})_{12}+ 6(\mathrm{pypz}) \mathrm{H} \rightarrow \\
& 3\left[\mathrm{Ru}(\mathrm{CO})_{2}(\mathrm{pypz})_{2}\right](\mathbf{2})+3 \mathrm{H}_{2}+6 \mathrm{CO}
\end{aligned}
$$

The Ru complex $\mathbf{2}$ was isolated in $\sim 70 \%$ yield after conducting column chromatography and recrystallization. The electron impact mass spectrometry revealed molecular ion $\mathrm{M}^{+}$with the expected composition $\mathrm{C}_{20} \mathrm{H}_{10^{-}}$ $\mathrm{F}_{6} \mathrm{~N}_{6} \mathrm{O}_{2} \mathrm{Ru}$, providing evidence for the incorporation of two pyridyl pyrazolate ligands. For the respective reaction with the heavy congener $\mathrm{Os}_{3}(\mathrm{CO})_{12}$, due to its higher thermal stability and lower reactivity, the condensation reaction was best carried out using a technique of direct solid-state pyrolysis and employing a much longer reaction time of $\sim 2.5$ days. The osmium analogue complex $\left[\mathrm{Os}(\mathrm{CO})_{2}(\mathrm{pypz})_{2}\right]$ (3) was then obtained in $39 \%$ yield, after vacuum sublimation and recrystallization from a mixture of $\mathrm{CH}_{2} \mathrm{Cl}_{2}$ and hexane at room temperature.

(13) (a) Liu, S.-F.; Wu, Q.; Schmider, H. L.; Aziz, H.; Hu, N.-X.; Popovic, Z.; Wang, S. J . Am. Chem. Soc. 2000, 122, 3671. (b) Liu, Q.; Mudadu, M. S.; Schmider, H.; Thummel, R.; Tao, Y.; Wang, S. Organometallics 2002, 21, 4743.

(14) (a) J ohnson, B. F. G. Inorg. Chim. Acta 1986, 115, L39. (b) Bogdan, P. L.; Weitz, E. J . Am. Chem. Soc. 1990, 112, 639. 


$$
\begin{aligned}
\mathrm{Os}_{3}(\mathrm{CO})_{12}+ & 6(\mathrm{pypz}) \mathrm{H} \rightarrow \\
& 3\left[\mathrm{Os}(\mathrm{CO})_{2}(\mathrm{pypz})_{2}\right](3)+3 \mathrm{H}_{2}+6 \mathrm{CO}
\end{aligned}
$$

For Os complex $3,{ }^{1} \mathrm{H},{ }^{13} \mathrm{C}$, and ${ }^{19} \mathrm{~F}$ NMR studies confirmed the presence of 2-pyridyl pyrazolate and $\mathrm{CO}$ ligands in an expected ratio of 2:2 within the ligand sphere. An extension of preparing analogues of this Os complex is available by the variation of substituents on the 2-pyridyl pyrazolate ligand. This modification gave two additional derivative complexes, $\left[\mathrm{Os}(\mathrm{CO})_{2}\left({\left.\text { pypz } 2)_{2}\right]}_{2}\right.\right.$ (4) and $\left[\mathrm{Os}(\mathrm{CO})_{2}\left(\mathrm{pypz}_{2}\right)_{2}\right]$ (5), pypz2 = 3-pentafluoroethyl-5-(2-pyridyl) pyrazolate and pypz3 = 3-trifluoromethyl-5-(4-methyl-2-pyridyl) pyrazolate. Their corresponding analytical and spectroscopic data as well as detailed spectral assignments are shown in the Experimental Section.

All three Os(II) complexes 3-5 show excellent thermal stability at temperatures $\sim 20{ }^{\circ} \mathrm{C}$ as well as good photochemical stability upon exposure to intensive UV irradiation. Another unique feature is that the introduction of a stronger electron-withdrawing substituent, such as $\mathrm{CF}_{3}$ or $\mathrm{C}_{2} \mathrm{~F}_{5}$, at the $\mathrm{C}(3)$ position of the pyrazole segment is indispensable to the successful preparation of the more robust third-row Os metal complexes. In this study, numerous attempts have been made to synthesize other $\left[\mathrm{Os}(\mathrm{CO})_{2}(\mathrm{pypz})_{2}\right]$ analogues based on methyl, tertbutyl, and phenyl substitution at the $\mathrm{C}(3)$ position, but unfortunately all failed, demonstrating the feasibility of synthesizing 3-5 mainly due to the greater reactivity of perfluoroalkyl-substituted pyrazole ligands.

3.2. Structural Characterization. Single-crystal $X$-ray diffraction studies were carried out to resol ve the exact molecular structures. As depicted in Figure 2, the structure of $\mathbf{2}$ shows an octahedral coordination around the Ru atom. The 2-pyridyl pyrazol ate ligands form fivemembered chelate ring structures with the pyrazolate nitrogen atoms $N(2)$ and $N(5)$ located at trans dispositions, while the cis carbonyl fragments occupy the sites trans to the 2-pyridyl nitrogen atoms $N(1)$ and $N(4)$, which are also arranged in the cis $(\mathrm{N}, \mathrm{N})$ fashion. The coordination sequence of the pairs of donor centers $\mathrm{CO}$, $\mathrm{N}_{\mathrm{py}}$, and $\mathrm{N}_{\mathrm{pz}}$ determines the observed cis-cis-trans conformation. The corresponding bond length and bond angle data are listed in Table 2. Metal-ligand bond angles found in this complex are within the range expected for typical octahedral Ru(II) complexes. The major deviation from a perfect octahedral coordination is caused by the smaller bite angles observed for the 2-pyridyl pyrazolate chelating interactions $(\angle \mathrm{N}(1)-\mathrm{Ru}-$ $\mathrm{N}(2)=77.5(1)^{\circ}$ and $\left.\angle \mathrm{N}(4)-\mathrm{Ru}-\mathrm{N}(5)=77.1(1)^{\circ}\right)$, while the overall ligand arrangement in complex 2 resembles that of the structurally characterized $\mathrm{Ru}(\mathrm{II})$ complexes such as $\left[\mathrm{Ru}(\mathrm{CO})_{2}(\mathrm{hfac})_{2}\right],{ }_{15}^{15}\left[\mathrm{Ru}(\mathrm{CO})_{2}\right.$ (1,2-naphthoquinone 1-oximate $\left.)_{2}\right],{ }^{16}$ and $\left[\mathrm{Ru}(\mathrm{CO})_{2} \text { (2-pyridyl carboxylate) }\right)_{2}{ }_{1},{ }^{17}$ possessing a pair of cis carbonyl ligands and the donor atoms of the bidentate chelates located in the remainder of the coordination sites. Moreover, the pyrazolate nitrogen atoms exhibit a much stronger donor interaction with the $\mathrm{Ru}(\mathrm{II})$ center than the dative interaction

(15) Lee, F.-J .; Chi, Y.; Hsu, P.-F.; Chou, T.-Y .; Liu, C.-S.; Peng, S.M.; Lee, G.-H. Chem. Vap. Deposition 2001, 7, 99.

(16) Lee, K. K.-H.; Wong, W.-T. J . Chem. Soc., Dalton Trans. 1997, 2987.

(17) Xu, L.; Sasaki, Y. J . Organomet. Chem. 1999, 585, 246.

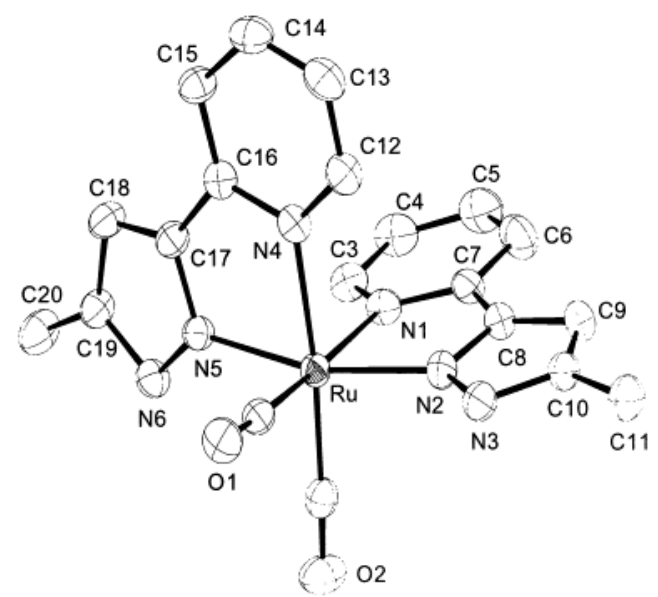

Figure 2. ORTEP diagram of complex 2 with thermal ellipsoids shown at $30 \%$ probability; the fluorine atoms of the $\mathrm{CF}_{3}$ substituent on the pyrazolate fragments were removed for clarity.

from the 2-pyridyl nitrogen atoms. Evidence for this viewpoint is provided by the significantly shorter $\mathrm{Ru}-$ $\mathrm{N}(\mathrm{pz})$ bond distances $(\mathrm{Ru}-\mathrm{N}(2)=2.058(3) \AA$ and $\mathrm{Ru}-$ $\mathrm{N}(5)=2.047(3) \AA)$ versus those of the $\mathrm{Ru}-\mathrm{N}(\mathrm{py})$ distances $(R u-N(1)=2.136(3) \AA$ and $R u-N(4)=2.140$ (3) $\AA$ ). This deviation could result from a combination of (i) the stronger donor character of the pyrazolate nitrogen atoms due to its -1 charge nature and (ii) the relatively stronger trans influence exerted by the carbonyl ligands, which significantly weaken the $N(p y) \rightarrow R u$ dative interaction. In good agreement with this postulation, the long Ru-N (py) distances in complex $\mathbf{2}$ compare well with those observed in the related $\mathrm{Ru}(\mathrm{II})$ metal complexes such as [Ru(CO)(py)(TTP)] (2.193(4) Å), TTP $=$ tetraphenylporphinate) and $\left[\mathrm{Ru}(\mathrm{bpy})(\mathrm{CO})_{2} \mathrm{Cl}_{2}\right](2.10$ (1) $\AA),{ }^{18}$ showing the pyridine group located trans to the strong $\pi$-accepting carbonyl ligand.

A perspective view of the Os complex $\mathbf{3}$ together with the atomic numbering scheme is illustrated in Figure 3 , and selected bond lengths and angles are listed in Table 3. To our surprise, the coordination environment around the Os atom, which shows an ideal $\mathrm{C}_{2}$ symmetry, displays a notable distinction from that established for the previous $\mathrm{Ru}$ analogue $\mathbf{2}$. This is revealed by the detection of pyrazolate nitrogen atoms $N(1)$ and $N(1 A)$ being located at the position trans to the cis-oriented carbonyl ligands. Although this subtle change of ligand orientation on the ligand sphere has had almost no effect on the chelate bite angle, of. $\angle \mathrm{N}(1)-\mathrm{Os}(1)-\mathrm{N}(2)$ $=77.3(1)^{\circ}$, the nitrogen to osmium dative bonding interaction has turned substantially stronger, as is evident from the observation of unusually short Os-N distances $(\operatorname{Os}(1)-\mathrm{N}(1)=2.013(3) \AA$ and Os(1) $-\mathrm{N}(2)=$ $2.087(3) \AA$ ). The latter is very close to the Os $-\mathrm{N}(\mathrm{bpy})$ distances $(2.036-2.076 \AA$ ) observed in the related Os(II) complexes such as $\left[\mathrm{Os}(\mathrm{bpy})_{2}(\mathrm{Cl})(\mathrm{NCMe})\right]\left[\mathrm{PF}_{6}\right]$ and $\left[\mathrm{Os}(\mathrm{bpy})_{3}\right]\left[\mathrm{PF}_{6}\right]_{2 .}{ }^{19}$ The results may be rationalized by the fact that the third-row transition metal elements tend to have stronger ligand-to-metal interactions com-

(18) (a) Little, R. G.; I bers, J . A. J . Am. Chem. Soc. 1973, 95, 8583 (b) Haukka, M.; Kiviaho, J .; Ahlgren, M.; Pakkanen, T. A. Organome tallics 1995, 14, 825.

(19) (a) Constable, E. C.; Raithy, P. R.; Smit, D. N. Polyhedron 1989, 8, 367. (b) Demadis, K. D.; Meyer, T. J .; White, P. S. Inorg. Chem. 1998, 37, 3610. 


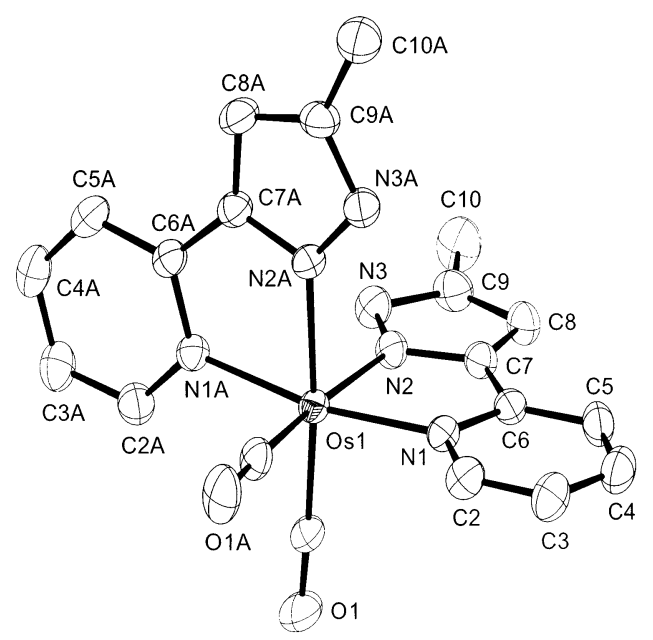

Figure 3. ORTEP diagram of complex $\mathbf{3}$ with thermal ellipsoids shown at $30 \%$ probability; the fluorine atoms of the $\mathrm{CF}_{3}$ substituent on the pyrazolate fragments were removed for clarity.

pared with their second-row congeners. In addition, the -1 charged pyrazolate nitrogen atoms are located at the positions trans to the good $\pi$-acceptor $\mathrm{CO}$ ligand, which can effectively remove the excess charge density that may accumulate at the metal center. As a result, the $\mathrm{N}(\mathrm{pz}) \rightarrow$ Os dative interaction is strengthened, resulting in a reduction of bond distances. I R $v(\mathrm{CO})$ spectral studies of Os complex 3 in $\mathrm{CH}_{2} \mathrm{Cl}_{2}$ solution revealed two CO stretching bands at 2043 and $1973 \mathrm{~cm}^{-1}$, which are at lower frequencies than those (2076 and $2017 \mathrm{~cm}^{-1}$ ) observed for the respective Ru complex $\mathbf{2}$. The results are in good agreement with the delineation, suggesting the occurrence of a stronger $\pi$-acceptor interaction within the Os complexes. For comparison, a reverse trend was observed in their parent carbonyl complexes, in which the $v(\mathrm{CO})$ stretching bands of $\mathrm{Os}_{3}(\mathrm{CO})_{12}$ occurred at least $4-8 \mathrm{~cm}^{-1}$ higher in frequency than those of the $\mathrm{Ru}_{3}(\mathrm{CO})_{12}$ counterpart. ${ }^{20}$

3.3. Photophysical Properties. Figure 4 shows UV-visible absorption spectra of the 2-pyridyl pyrazolate complexes in acetonitrile. F or metal complexes 2 and 3, the dominant absorption bands in the 245-316 $\mathrm{nm}$ region were ascribed to the ligand-centered $\pi-\pi^{*}$ transitions. These spectral assignments were based on the absorption spectra of the related boron complex $\mathbf{1}$, as well as the corresponding free ligands (shown later in Figure 6). Attempts have al so been made to examine any lower energy absorption at > $360 \mathrm{~nm}$, presumably associated with typical MLCT transitions. However, no absorption bands could be resolved in the region of 380$700 \mathrm{~nm}$, suggesting that all MLCT transitions in complexes $\mathbf{2}$ and $\mathbf{3}$ are hidden in the UV region of the strong intra-ligand $\pi-\pi^{*}$ transitions (vide infra). Absorption spectra similar to those for complex $\mathbf{3}$ were observed for Os(II ) complexes 4 and 5, and their relevant photophysical data are listed in Table 4.

Taking complex 3 as a prototype, a typical emission spectrum of 5-(2-pyridyl) pyrazolate Os complexes is depicted in Figure 4. Despite the diffusive $\mathrm{S}_{0} \rightarrow \mathrm{S}_{1}(\pi-$ $\left.\pi^{*}\right)$ absorption band, the corresponding emission spec-

(20) (a) J ohnson, B. F. G.; Lewis, J . Inorg. Synth. 1972, 13, 92. (b) Battiston, G. A.; Bor, G.; Dietler, U. K.; Kettle, S. F. A.; Rossetti, R.; Sbrignadello, G.; Stanghellini, P. L. I norg. Chem. 1980, 19, 1961.

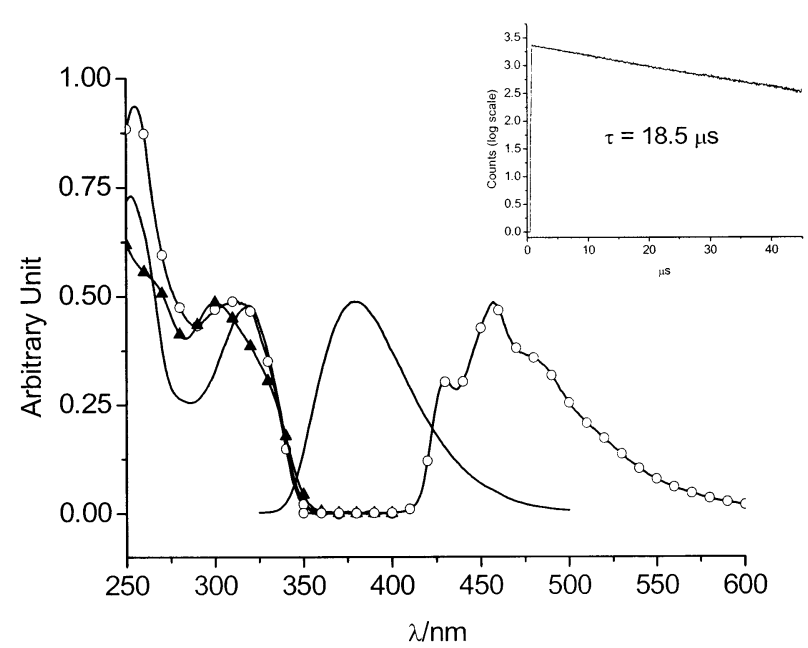

Figure 4. Room-temperature UV-vis absorption and emission spectra of the pypz complexes 1 (-), 2 (- $\mathbf{-}-)$, and 3 (-O-) in $\mathrm{CH}_{3} \mathrm{CN}$, in which the emission of $\mathbf{2}$ is not detectable. Inset: Relaxation dynamics of emission for $\mathbf{3}$ in room-temperature degassed $\mathrm{CH}_{3} \mathrm{CN}$.

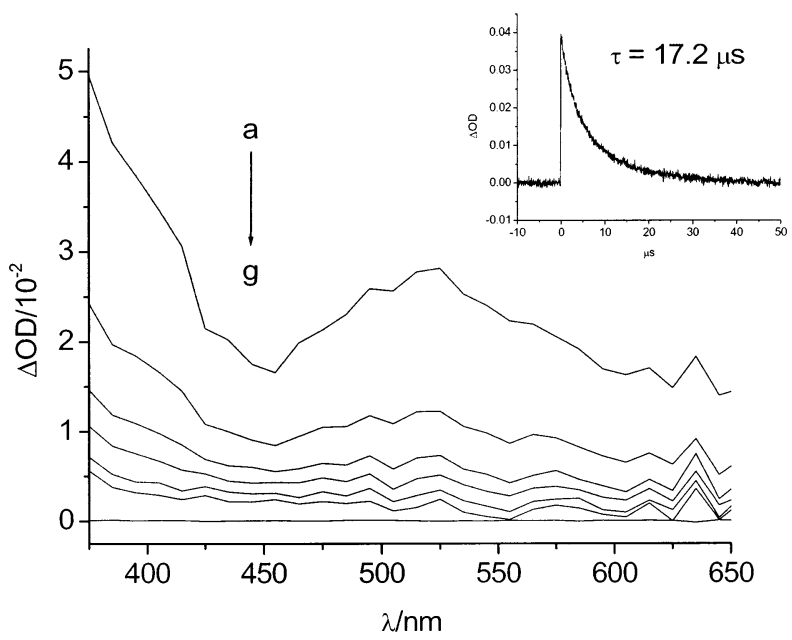

Figure 5. Temporal evolution of transient absorption spectra for complex 3 at a delay time of (a) 0.36 , (b) 6 , (c) 11.7, (d) 17.4 , (e) 28.8, and (f) $40 \mu \mathrm{s}$ in degassed $\mathrm{CH}_{3} \mathrm{CN}$; (g) transient absorption spectrum of $\mathbf{3}$ acquired at a delay time of $2 \mu \mathrm{s}$ in aerated $\mathrm{CH}_{3} \mathrm{CN}$. I nset: Decay profile of the transient signal at $550 \mathrm{~nm}$ (in degassed $\mathrm{CH}_{3} \mathrm{CN}$ ). $\lambda_{\text {ex }}: 355$ $\mathrm{nm}$.

trum exhibits a distinct vibronic feature with peak maxima at 430,457 , and $\sim 480 \mathrm{~nm}$ in $\mathrm{CH}_{3} \mathrm{CN}$. The luminescence intensity is linearly proportional to the increase of concentrations, eliminating the emissions associated with any high-order aggregation. The entire emission band originating from a common ground-state species is ascertained by the same fluorescence excitation spectra throughout the monitored wavelengths of 420-600 $\mathrm{nm}$. The excitation spectra, within the range of experimental error, are also effectively identical to the absorption spectrum, indicating that the entire emission results from a common Franck-Condon excited state. From comparison of the corresponding absorption and emission spectra, we can make several remarks regarding complex $\mathbf{3}$. First of all, there is a significantly large energy gap of $\sim 4000 \mathrm{~cm}^{-1}$ for the $0-0$ vibronic onsets between absorption and emission spectra. In comparison, complex $\mathbf{1}$ exhibits an emission band 


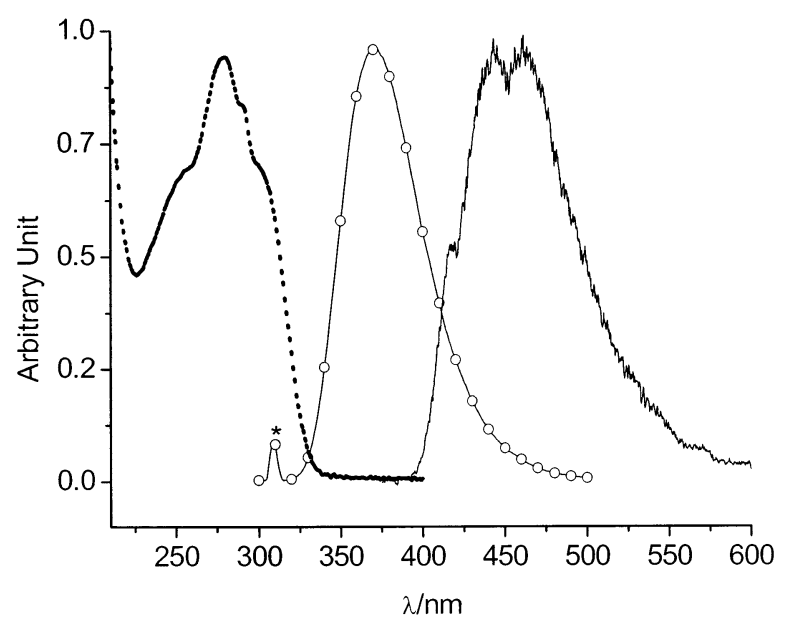

Figure 6. (a) Room-temperature UV-vis absorption (- - ) and fluorescence (-O-) spectra of pypz anionic species (pypzK) in $\mathrm{CH}_{3} \mathrm{CN}$ containing $1 \mathrm{M} \mathrm{KOH}$. (b) Phosphorescence spectrum of pypz anion (-) in the $77 \mathrm{~K}$ solid $\left(\mathrm{CH}_{3-}\right.$ $\mathrm{CN})$ matrix acquired at a delay time of $1 \mu \mathrm{s}$. * denotes Raleigh scattering.

Table 4. Photophysical Properties of Complex 1-5 in Room-Temperature $\mathrm{CH}_{3} \mathrm{CN}^{\mathrm{a}}$

\begin{tabular}{ccclc}
\hline complex & absorption $\left(\mathrm{S}_{0}-\mathrm{S}_{1}\right)$ & emission & \multicolumn{1}{c}{ Q.Y. } & \multicolumn{1}{c}{$\tau_{\text {obs }}$} \\
\hline $\mathbf{1}$ & $319(\epsilon=14250)^{\mathrm{b}}$ & 380 & 0.88 & $6.4 \mathrm{~ns}$ \\
$\mathbf{2}$ & $300(\epsilon=14000)$ & $462^{\mathrm{c}}$ & & $50.5 \mu \mathrm{S}^{\mathrm{c}}$ \\
$\mathbf{3}$ & $311(\epsilon=16500)$ & 430 & 0.14 & $18.5 \mu \mathrm{s}$ \\
& & 457 & & \\
& & 480 & & \\
$\mathbf{4}$ & $314(\epsilon=16120)$ & 430 & $8.69 \times 10^{-2}$ & $13.4 \mu \mathrm{s}$ \\
& & 455 & & \\
$\mathbf{5}$ & $306(\epsilon=17200)$ & 480 & & \\
& & 428 & $4.06 \times 10^{-2}$ & $6.3 \mu \mathrm{s}$
\end{tabular}

480

a All samples were degassed via three freeze-pump-thaw cycles. ${ }^{\mathrm{b}}$ Units in $\mathrm{M}^{-1} \mathrm{~cm}^{-1}$. ${ }^{\mathrm{c}}$ Data were obtained at $77 \mathrm{~K}$.

maximized at $380 \mathrm{~nm}$, in which the $0-0$ onset overlaps with that of the $S_{0} \rightarrow S_{1}\left(\pi-\pi^{*}\right)$ absorption band. Second, the Stokes shift, defined as the peak-to-peak frequency between absorption and emission, is as large as 9000 $\mathrm{cm}^{-1}$. Since the emission peak wavelength revealed a slightly hypsochromic shift from cyclohexane (460 nm) to acetonitrile (457 nm), the anomal ously large Stokes shifted emission resulting from the solvent dipolar relaxation also can be discarded. Alternatively, these observations, in combination with the relaxation dynamics, $\mathrm{O}_{2}$ quenching effect, and transient absorption spectra elaborated as follows, lead us to propose that the emission, more plausibly, originates from a triplet manifold.

The inset of Figure 4 shows the relaxation dynamics of emission for complex $\mathbf{3}$ in room-temperature degassed $\mathrm{CH}_{3} \mathrm{CN}$. The logarithm plot of the time-dependent emission intensity is apparently linear and thus can be well fitted straightforwardly by the first-order relaxation dynamics. A best linear least-squares fit gave the lifetime of complex $\mathbf{3}$ as $18.3 \mu \mathrm{s}$. The same method was applied for complexes $\mathbf{4}$ and $\mathbf{5}$ with corresponding lifetimes of 13.4 and $6.3 \mu \mathrm{s}$, respectively (see Table 4). U pon aeration of the solution, the lifetime of complex $\mathbf{3}$ was drastically reduced to $310 \mathrm{~ns}$, accompanied by the decrease of the steady-state emission intensity (not shown here). Taking an $\mathrm{O}_{2}$ concentration of $1.9 \times 10^{-3}$
$\mathrm{M}$ in the aerated $\mathrm{CH}_{3} \mathrm{CN}$ solvent, ${ }^{21}$ a quenching rate constant of $\sim 1.7 \times 10^{9} \mathrm{M}^{-1} \mathrm{~s}^{-1}$ was thus deduced, which is nearly $1 / 9$ of the diffusion-controlled rate of $1.8 \times 10^{10}$ $\mathrm{M}^{-1} \mathrm{~S}^{-1}$ calculated from the Stokes-Einstein equation ${ }^{22}$ in $\mathrm{CH}_{3} \mathrm{CN}$. The result is consistent with the $\mathrm{O}_{2}$ quenching triplet state according to the theory of electronexchange type energy transfer expressed as

$$
\mathrm{T}+{ }^{3} \mathrm{O}_{2} \stackrel{\mathrm{k}_{\mathrm{O}_{2}}}{\longrightarrow} \mathrm{S}_{0}+{ }^{1} \mathrm{O}_{2}
$$

The overall spin must be conserved upon forming a collisional complex. Accordingly, the possibility of each collision generating ${ }^{1} \mathrm{O}_{2}$ is statistically $1 / 9$.

Another key support for the triplet-state population was rendered by the transient absorption experiment. Figure 5 shows the temporal spectral evolution of the transient absorption for complex $\mathbf{3}$, consisting of a wellresolved band maximized at $520 \mathrm{~nm}$ and the other one at $<370 \mathrm{~nm}$ that was irresolvable due to spectral interference with the ground-state absorption. As shown in the inset of Figure 5, a plot of the transient absorbance versus the delay time reveals a biexponential feature. In other words, the logarithm plot of the transient absorbance does not reveal a straight line (not shown here) and thus cannot be simply fitted by firstorder decay kinetics. With an increase in the laser intensity the non-single-exponential decay behavior became more obvious, indicating that the bimolecular quenching process, i.e., the triplet-triplet annihilation, plays a role in the relaxation dynamics. In a degassed solution where $\mathrm{O}_{2}$ has been completely removed, the decay pathways of a triplet-state species incorporating triplet-triplet annihilation can be expressed as

$$
\begin{gathered}
\mathrm{T} \stackrel{\mathrm{k}_{\mathrm{T}}}{\longrightarrow} \mathrm{S}_{0} \\
\mathrm{~T}+\mathrm{T} \stackrel{\mathrm{k}_{\mathrm{TT}}}{\longrightarrow} \mathrm{S}_{1}+\mathrm{S}_{0}
\end{gathered}
$$

By solving the associated kinetic equations, the timedependent triplet-state concentration $[\mathrm{T}]$ is depicted as

$$
[T]=\frac{k_{T}[T]_{0}}{\left(k_{T}+k_{T T}[T]_{0}\right) e^{k_{T} t}-k_{T T}[T]_{0}}
$$

where $[T]_{0}$ is the concentration of the triplet-state species prepared right after the laser excitation. Under the experimental condition of $1 \mathrm{~cm}$ excitation path length, the time-dependent transient absorbance $\Delta \mathrm{A}$ should theoretically be proportional to $[T]$ by $\Delta \mathrm{A}=\epsilon_{\mathrm{TT}^{-}}$ $[T]$, where $\epsilon_{\mathrm{TT}}$ is the extinction coefficient of the triplettriplet absorption. Letting $b=k_{T T} / \epsilon_{\mathrm{TT}}$, eq 3 can thus be rewritten as

$$
\Delta \mathrm{A}=\frac{\mathrm{k}_{\mathrm{T}} \Delta \mathrm{A}_{0}}{\left(\mathrm{k}_{\mathrm{T}}+\mathrm{b} \Delta \mathrm{A}_{0}\right) \mathrm{e}^{\mathrm{k}_{\mathrm{T}} \mathrm{t}}-\mathrm{b} \Delta \mathrm{A}_{0}}
$$

where $\Delta \mathrm{A}_{0}$ is the signal intensity right after the laser pulse. The decay of the transient absorbance depicted in the inset of Figure 5 was well fitted by eq 4, and the

(21) Murov, S. L.; Carmichael, I.; Hug, G. L. Handbook of Photochemistry, 2nd ed.; Marcel Dekker: New York, 1993.

(22) For example, see: Birks, J. B. Photophysics of Aromatic Molecules; Wiley: New York, 1970; p 313. 
lifetime was deduced to be $17.2 \mu \mathrm{s}$, which, within the range of experimental error, is identical to the one (18.3 $\mu \mathrm{s})$ derived from the emission relaxation dynamics. N ote that due to the rather small triplet-state population, the triplet-triplet annihilation is negligible in the emission study using the time-correlated single-photon-counting technique. Thetransient absorption originating from the triplet-triplet transition was also confirmed by its nearly $1 / 9$ diffusion-controlled $\mathrm{O}_{2}$ quenching rate, as indicated by the negligible transient absorbance in aerated solution acquired at a delay time of, for example, $2 \mu \mathrm{s}$ (see Figure $5 \mathrm{~g}$ ). Similar emission properties such as spectral features, long lifespan $(>\mu \mathrm{S})$, and $\mathrm{O}_{2}$ quenching dynamics were observed for complexes $\mathbf{4}$ and $\mathbf{5}$, and the corresponding photophysical data are listed in Table 4.

Upon unambiguously determining the dominant emission in Os(II) complexes 3-5 to be of phosphorescent nature, our next task focuses on the origin of the phosphorescence. The appearance of phosphorescence with vibronic progression has provided a clue to the ${ }^{3} \pi-$ $\pi^{*}$ configuration, which plausibly originates from the ligand-center chromophore. To validate this assignment, we have carried out a series of comparative studies focusing on the photophysics of the ligand 5-(2-pyridyl) pyrazole (pypzH). Due to the deprotonation of pypzH in forming the corresponding Os(II) complex, a fair comparison should be with its anion species. Figure 6a shows the room-temperature absorption and emission spectra of the pypz anion (pypzK), in which the roomtemperature emission consists of a unique, normal Stokes-shifted fluorescence maximized at $372 \mathrm{~nm}\left(\tau_{\mathrm{f}} \approx\right.$ $4.38 \mathrm{~ns})$. No longer wavelength emission ascribed to phosphorescence could be resolved in room-temperature $\mathrm{CH}_{3} \mathrm{CN}$. Figure $6 \mathrm{~b}$ shows the emission of pypz anion in a $77 \mathrm{~K}$ solid matrix $\left(\mathrm{CH}_{3} \mathrm{CN}\right)$ acquired after a delay time of $1 \mu \mathrm{s}$ to el iminate the fluorescence interference. Apparently, a structured phosphorescence maximized at $\sim 460 \mathrm{~nm}$ was resolved, of which the spectral feature resembles that observed in complex $\mathbf{3}$. This result unambiguously indicates that the room-temperature phosphorescence in the title Os complexes originates from the ligated pypz chromophore. N ote that the main difference in phosphorescence properties between the free pypz anion and complex 3 lies in the $\tau_{\mathrm{p}}>1 \mathrm{~s}$ phosphorescence lifetime (at $77 \mathrm{~K}$ ) in the pypz anion, which is longer than that in complex 3 by $\sim 5$ orders of magnitude, manifesting the unusually strong spin-orbit coupling in complex 3. Similar phosphorescence properties can be ascribed to compounds $\mathbf{4}$ and $\mathbf{5}$. However, due to the relatively small radiative decay rate, the radiationless decay pathway induced by methyl (or $\mathrm{CF}_{3}$ ) torsional motion may play a key role in the quenching of phosphorescence. Accordingly, among compounds 3-5 the smallest phosphorescence quantum yield for complex $\mathbf{5}$ can be rationalized because of its bearing two methyl-like rotors $\left(-\mathrm{CH}_{3}\right.$ and $\left.-\mathrm{CF}_{3}\right)$. Likewise, the phosphorescence quantum yield of complex 4 with $\mathrm{C}_{2} \mathrm{~F}_{5}$ substituent, being smaller than that of complex $\mathbf{3}$ with $\mathrm{CF}_{3}$, is plausibly due to the more torsional degrees of freedom in $\mathrm{C}_{2} \mathrm{~F}_{5}$.

Another key comparison was made via the study of complex 2, containing a Ru(II) metal ion. Intriguingly, under the detection limit of $\sim 10^{-5}$ quantum efficiency no detectable emission can be resolved in room-temperature, degassed $\mathrm{CH}_{3} \mathrm{CN}$ solution. The transient absorption measurement was also performed for Ru complex 2. Under the same experimental conditions, a transient absorbance of $\sim 1 / 10$ that of Os complex 3 with similar spectral features ( $\lambda_{\max } \approx 520 \mathrm{~nm}$, not shown here) was obtained for Ru complex $\mathbf{2}$. U pon cooling to $77 \mathrm{~K}$, a weak, structural phosphorescence maximized at $\sim 460 \mathrm{~nm}\left(\tau_{\mathrm{p}}\right.$ $\approx 50 \mu \mathrm{s}$ ) was resolved, and it clearly possesses a pypz $3 \pi-\pi^{*}$ character, confirming the deactivation of the initial ${ }^{1} \pi-\pi^{*}$ excited states to the lowest ${ }^{3} \pi-\pi^{*}$ triplet state via intersystem crossing. However, as the ligand field strength associated with the second-row Ru(II) atom is much weaker than that of the third-row Os(II) analogues, the $d-d$ levels of the Ru complexes would be significantly lowered and become thermally accessible from the ${ }^{3} \pi-\pi^{*}$ level, as the latter is somewhat independent of the variation of the central metal atom. ${ }^{23}$ In such cases, the ${ }^{3} \mathrm{LC}\left(\pi-\pi^{*}\right)$ state is expected to internally relax to the $d-d$ states through a thermally activated process, followed by a rapid, dominant radiationless pathway to give negligible room-temperature phosphorescence. In contrast, due to the greater ligand field strength, the $d-d$ levels in Os complexes are thermally inaccessible from the ${ }^{3} \mathrm{LC}\left(\pi-\pi^{*}\right)$ state, so the competing radiationless deactivation no longer plays a major role in quenching the ${ }^{3} \mathrm{LC}\left(\pi-\pi^{*}\right)$ state, resulting in a strong intra-ligand ${ }^{3} \pi-\pi^{*}$ phosphorescence in the room-temperature solution phase.

\section{Discussion}

Based on the above results, the photophysical properties of these new series of $\left[\mathrm{M}(\mathrm{CO})_{2}(\mathrm{pypz})_{2}\right]$ complexes may be rationalized through their structural uniqueness. The strong $\pi$-accepting ancillary $\mathrm{CO}$ ligands are capable of removing the electron density from the cationic metal center and hence lead to a higher oxidation potential for the current $\mathrm{M}(\mathrm{II})$ metal center as well as the destabilization of the M(III) metal center. This electron-withdrawing characteristic, being much greater than those observed for the phosphine and arsine chelates, ${ }^{24}$ would substantially increase the relative energy of the typical low-lying metal-to-ligand (Os$\left.(\mathrm{d} \pi) \rightarrow\left(\pi^{*}\right)\right)$ charge transfer transition and make the pypz ligand-centered $\pi-\pi^{*}$ energy level the lowest singlet excited state. Evidence has been found from the electronic absorption spectroscopy, in which the MLCT transitions were obscure and possibly hidden inside the strong ligand-centered $\pi-\pi^{*}$ transition. It should be noted that the relatively different ligand-versus-CO orientation, to a certain extent, seems to influence the spectral properties as well. For example, Os(II) complex 3 shows a bathochromic shift of the absorption spectrum and relatively higher peak extinction coefficient (see Table 4) than that of Ru complex $\mathbf{2}$, consistent with different extents of the $\mathrm{N}(\mathrm{pz})$-metal dative interaction.

Upon electronic excitation, the strong spin-orbit coupling expected for Os(II) compl exes 3-5 would lead to an efficient intersystem crossing from the singlet excited state to the triplet manifold of the ligands. The

(23) Demas, J . N.; DeGraff, B. A. Anal. Chem. 1991, 63, 829A.

(24) (a) J ohnson, S. R.; Westmoreland, T. D.; Caspar, J . V.; Barqawi, K. R.; Meyer, T. J. Inorg. Chem. 1988, 27, 3195. (b) Kober, E. M. Caspar, J . V.; Sullivan, B. P.; Meyer, T. J . Inorg. Chem. 1988, 27, 4587. 
fast rate of intersystem crossing is supported by the lack of the 2-pyridyl pyrazolate fluorescence in the region of $370 \mathrm{~nm}$, as well as the system-response-limit rise time for the phosphorescence ( $<200 \mathrm{ps})$. The strong spinorbit coupling, to a great extent, also removes the spinforbidden nature of the $\mathrm{T}_{1} \rightarrow \mathrm{S}_{0}$ radiative relaxation. As a result, a dominant bluegreen phosphorescent emission can be clearly observed in the room-temperature solution phase. For complex $\mathbf{3}$, the phosphorescence yield $\left(\Phi_{\mathrm{p}}\right)$ was measured to be 0.14 in the degassed $\mathrm{CH}_{3-}$ $\mathrm{CN}$ solvent. Assuming unitary efficiency for the intersystem crossing and neglecting the emission loss due to the triplet-tripl et annihilation, the $\mathrm{T}_{1} \rightarrow \mathrm{S}_{0}$ radiative decay rate constant $\mathrm{k}_{\mathrm{r}}$ can be derived from a relationship expressed as $\mathrm{k}_{\mathrm{r}}=\Phi_{\mathrm{p}} \mathrm{k}_{\mathrm{obs}}$, where $\mathrm{k}_{\mathrm{obs}}$ was measured to be $5.46 \times 10^{4} \mathrm{~s}^{-1}$. Accordingly, a $\mathrm{k}_{\mathrm{r}}$ value of $7.64 \times 10^{3}$ $\mathrm{s}^{-1}$ was deduced. In comparison to the $<1.0 \mathrm{~s}^{-1}$ phosphorescence radiative decay rate of the corresponding free ligand anion (i.e., pypz ${ }^{-}$anion), the vast enhancement of the $\mathrm{T}_{1} \rightarrow \mathrm{S}_{0}$ radiative decay rate through the spin-orbit coupling in complexes $\mathbf{3}-\mathbf{5}$ is supportive. On the other hand, as indicated by the lack of fluorescence and small $T_{1}-T_{n}$ transient absorbance, the rapid excitedstate relaxation in complex $\mathbf{2}$ seems to be dominated by two additional factors. First, the small difference in energy between the ${ }^{3} \pi-\pi^{*}$ and the metal-centered $d-d$ states would promote facile thermal deactivation through population of the d-d-states, followed by radiationless, vibrational relaxation. Second, the enhanced nonradiative decay observed in complex $\mathbf{2}$ may also be rationalized via a ligand orientation effect. This is evidenced by the fact that the spatial separation between the cis pyridyl fragments in complex 2, estimated by the centerto-center distance between two pyridyl ring systems, is approximately $4.76 \AA$. This would then promote the inter-ligand el ectron transfer and give a fast nonradiative decay process. In contrast, complex $\mathbf{3}$ shows pyridyl sites located at the trans disposition and having a much longer intramolecular spatial separation of $6.9 \AA$, so that the inter-ligand through-space interaction is less effective than that expected in complex $\mathbf{2}$. F urther support of this viewpoint is given by a recent study on the luminescent properties of a series of tris-cyclometalated Ir(III) complexes, which have shown an approximate 10fold reduction of the emission quantum efficiency for the meridional isomers with respect to the alternative facial isomers. ${ }^{25}$ It is suggested that the weaker $\mathrm{Ir}-\mathrm{C}$ bonds observed in the meridional isomers would provide a much greater bond distortion in the excited state, and this could be the more likely reason for the effective quenching of the phosphorescent emission.

\section{Conclusion}

In conclusion, we report the syntheses and characterization of a new family of thermal and photochemical robust Os(II) and Ru(II) metal complexes. While the respective boron complex $\mathbf{1}$ exhibits strong fluorescent emission derived from the singlet $\pi-\pi^{*}$ chromophore, our results clearly demonstrate for the first time that Os(II) complexes 3-5 are capable of displaying a highenergy ligand-centered phosphorescence in the roomtemperature solution phase, despite the fact that Os(II) complexes typically exhibit lower energy MLCT emission in the regions of green, red, and even infrared. The presence of strong $\pi$-accepting ancillary $\mathrm{CO}$ ligands as well as the Os(II) heavy atom effect makes the assynthesized pyridyl pyrazolate complexes unique in their photophysical properties. Taking into account knowledge that current interest in the blue and redcolor emitter has recently been focusing on the Ir(III) type of $\pi-\pi^{*}$ or MLCT phosphorescence, future applications of the title Os(II) complexes and/or relevant derivatives may be motivated by the strategy of using these complexes in the fabrication of organic lightemitting diodes (OLEDs). The strong metal-ligand bonding interaction and high phosphorescence quantum efficiencies with ca. microsecond lifetimes may improve OLED performance. Focus on this approach is currently in progress.

Acknowledgment. We thank the National Science Council of Tai wan for financial support (NSC 91-2119M-002-016 and NSC 91-2113-M-007-006).

Supporting Information Available: X-ray crystallographic file (CIF and PDF) for complexes $\mathbf{2}$ and $\mathbf{3}$. This material is available free of charge via the Internet at http://pubs.acs.org.

OM034037E

(25) Tamayo, A. B.; Alleyne, B. D.; Djurovich, P. I.; Lamansky, S.; Tsyba, I.; Ho, N. N.; Bau, R.; Thompson, M. E. J . Am. Chem. Soc. 2003, $125,7377$. 